\title{
AN ALTERNATIVE APPROACH TO THE DATING OF BUSINESS CYCLE: NONPARAMETRIC KERNEL ESTIMATION
}

\author{
Jitka Poměnková*
}

\begin{abstract}
:
The paper provides the methodological background for the Czech Republic business cycle dating process using an alternative approach. This approach is based on the mathematical principle of identification of extremes using estimates of derivations of time trend of the analysed time series, for which the nonparametric Gasser-Müller estimate is used. The presented methodological approach is applied on the real gross domestic product data sets, the total industry (excluding construction), the gross capital formation and the final consumption expenditure. The selected variables are taken from the national accounts system. The obtained results are compared to the widely used naive technique of business cycle dating written by Canova $(1998,1999)$ or Bonenkamp (2001). The presented new method specifies the identification of turning points in the business cycle dating process.
\end{abstract}

Keywords: Gasser-Müller estimate, business cycle, identification of turning points, stabilization policy

JEL Classification: E32, C14

\section{Introduction}

The fiscal or/and monetary policy has been recently used in the sense of its stabilization function. The stabilization function involves an attempt to minimize deviations of economic activity from its potential or to minimize inflation deviations from the explicit inflation target. Keynes assumes that a decentralized market system does not automatically generate full employment and stable prices. In advanced industrial countries if people tend to save more than their incomes growth and their private consumption decreases, the investments have to rise in order to maintain full employment. The policy makers should pursue the stabilization policies. The main problem of stabilization policy application is to determine whether the time has come to act. This problem, called recognition lag, is based on statistical information which is not able to distinguish between random fluctuations and fundamental shifts in economic trends. The presented methodological approach upgrades the business cycle turning points identification.

* Jitka Poměnková, Faculty of Business Economics, Mendel University Brno, Zemědělská 1, 613 00 Brno, CR (pomenka@mendelu.cz). The results introduced in the paper are supported by the Research Intent No. MSM 6215648904 with the title "The Czech Economy in the Process of Integration and Globalisation, and the Development of Agricultural Sector and the Sector of Services under the New Conditions of the Integrated European Market" 
The definition of business cycle given by Burns and Mitchel (1946) says that "Business cycles are a type of fluctuations found in the aggregate economic activity of nations that organises their work mainly in business enterprises: a cycle consists of expansion occurring at the same time in many economic activities, followed by similarly general recessions, contractions, and revivals which merge into the expansion phase of the next cycle; in duration business cycles vary from more than one year to ten to twelve years; they are not divisible into shorter cycles of similar character with amplitudes approximating their own." There exist two approaches to the business cycle. The first one is called the classical business cycle and is based on a fluctuation in the level. The second one is known as the growth cycle and is based on a fluctuation around a trend. The dating of cycles is usually done by identifying turning points. The time period is usually too short; full of structural breaks and shocks. On the other hand, the growth cycle, where turning points are characterized by changes relative to the trend, represents a more promising version of the business cycles (Artis et al., 2004). Various methods providing valuable results can be used in both approaches.

Deterministic, stochastic or combination models are commonly used in the time domain. Their applications assume the knowledge of time series distribution and satisfaction of conditions for their use. It is problematic to determine the distribution type in small sample size time series, like the quarterly data sets of post-transformation economies. Nonparametric estimates represent a different approach since they do not require the knowledge about the distribution. Compared to parametric methods, limitations of using nonparametric methods are rather smaller. The well known nonparametric estimates are the Nadaraya-Watson estimate, the local linear estimate, and the Gasser-Müller (GM) estimate (Wand, Jones; 1995).

In order to implement the monetary or fiscal policy a more sophisticated methodology is required. The business cycle dating process includes not only fluctuations in the economic activity analysis but also the identification of turning points. To identify the moments when the economic activity is in the peak above the long-term trend or in the trough, it is essential to estimate the derivations of functions. The GM estimate is an efficient tool in estimating the derivation of functions and identifying the moments of peaks and troughs. Mathematically, using the GasserMüller estimates of the corresponding derivation orders of time series we can identify the maxima or minima in the analysed time series.

In the case of nonparametric estimates, it is difficult to produce a precise theoretical description of smoothers for a finite sample size. Therefore, it is important to make a simplification, which can be achieved in two ways. Firstly, the accuracy of a nonparametric smoothing method is evaluated as an infinite sample size. Secondly, we consider a smoother with a simple structure (the kernel smoother). As Härdle (1990) wrote, it would be a mistake to understand the asymptotical character of smoothers literally from the point of view of finite sample size. Nevertheless, if asymptotical reasons are applied sensitively they can provide meaningful ideas.

The presented paper provides the methodological background for an alternative approach in the business cycle dating process compared to the currently used methods. The concept is based on the mathematical principle of the extreme point identification using the estimates of time series trend derivations. For this purpose the nonparametric Gasser-Müller estimate is used. The presented methodology is demonstrated on 
real values and completed by the business cycle theory. The obtained results are compared to the widely used dating technique mentioned in Canova $(1998,1999)$ or Bonenkamp (2001) which is known as the naive dating technique. Presenting the new methodological approach allows us to specify the turning points in the business cycles and to improve the monetary or fiscal policy implementation efficiency.

\section{Methodology}

An alternative approach to the process of dating of business cycle is based on nonparametric kernel estimate. The basic process of proposed algorithm uses mathematical approach of identification of extreme points in the data set. That is, using an estimate of the first derivative of time trend we decide about the existence of stationary points, i.e. points in which the time trend has an extreme. Consequently, the second (or a higher order, if necessary) derivative of the time trend of given data, provides a decision about the type of extremes.

For this purpose the theoretical background of kernel estimates will follow. At first, the definition of kernel and smooth kernel used for the kernel estimation is done. Consequently, the Gasser-Müller estimate suitable for estimating derivations of the time trend is provided. Also, instruments for finding the optimum parameter, i.e. the bandwidth, is added. At the end, the naive rules written in Canova (1999) are mentioned.

Let the values $x$ be fixed design points chosen by an experimenter and let the real or simulated values $Y$ be dependent on the values $x$. The aim of regression analysis is to describe the relation between these two variables $(x, Y)$ and thus make a reasonable approximation of an unknown function $m$. This curve approximation is called smoothing. The regression formula describing the relation between $\left(x_{i}, Y_{i}\right)$, $i=1, \ldots n$ can be written in the following form

$$
Y_{i}=m\left(x_{i}\right)+\varepsilon_{i}, \quad i=1, \ldots, n,
$$

where $m$ is an unknown regression function, $x_{i}$ is a design point, $Y_{i}$ is a measurement, $\varepsilon_{i}$ are independent random variables for which the mean value is $E\left(\varepsilon_{i}\right)=0$ for $i=1, \ldots, n$ and the variance is $D\left(\varepsilon_{i}\right)=\sigma^{2}>0$ for $i=1, \ldots, n$ (Wand, Jones; 1995). The aim of the kernel smoothing is to find a suitable approximation $\hat{m}$ of the unknown function $m$. We will assume the design points $x_{i}$ to be equidistantly distributed over the interval $[0,1]$.

Definition 1. Let $v, k$ be nonnegative even integers such that $0 \leq v<k, k>2$. Let $K \in \operatorname{Lip}[-1,1]$, support $(\mathrm{K}) \subseteq[-1,1]$. Let the following moment conditions be satisfied

$$
K(-1)=K(1)=0 \text {, }
$$

$$
\int_{-1}^{1} x^{j} K(x) d x=\left\{\begin{array}{cc}
0 & 0 \leq j<k \\
(-1)^{v} v ! & j=v \\
\beta_{k} \neq 0 & j=k
\end{array}\right.
$$


Then we say that the kernel $K$ is of the order $(v, \mathrm{k})$ and write $K \in S_{v, k}{ }^{0}$. These kernels are used for an estimation of the regression function (Müller, 1988).

Definition 2. Let $v, k$ be nonnegative integers such that $0 \leq v<k, \mu \geq 1$. Function, $K \in C^{\mu}[-1,1]$, support $(K) \subseteq[-1,1]$ which satisfies the conditions

i)

$$
K^{(j)}(-1)=K(1)^{(j)}=0, \quad \mathrm{j}=0, \ldots, \mu-1
$$

ii)

$$
\int_{-1}^{1} x^{j} K(x) d x=\left\{\begin{array}{cc}
0 & 0 \leq j<k \\
(-1)^{v} v ! & j=v \\
\beta_{k} \neq 0 & j=k
\end{array}\right.
$$

is called a kernel of smoothness $\mu$, order $(v, \mathrm{k})$ and we write $K \in S_{v, k}^{\mu}$. See Horová $(2000,2002)$ for details.

The kernel estimates can be generally expressed in the form $\hat{m}=\sum_{i=1}^{n} W_{i}(x ; h) Y_{i}$, where $W_{i}(x, h)$ denotes a weight function, $h=h_{n}$ is a positive constant called a bandwidth, $h<x<1-h, K \quad$ is a kernel. Let $K \in S_{0 \kappa}$, the set $K_{h}(\cdot)=\frac{1}{h} K\left(\frac{\cdot}{h}\right)$, $h \in(0,1)$. Notice that the bandwidth, the kernel and the order of the kernel have a big influence on the quality of the estimate.

The Gasser-Müller (GM) estimate of the unknown regression function $m$ can be defined as follows

$$
\bar{m}^{(v)}(x)=\sum_{i=1}^{n} Y_{i} \frac{1}{h^{v+1}} \int_{s_{i-1}}^{s_{i}} K\left(\frac{x-u}{h}\right) d u .
$$

The design points $x_{i} \in[0,1], i=1, \ldots, n$, are ordered according to their size and for points $s_{i}, i=0, \ldots, n$ holds $s_{0}=0, s_{i}=\left(x_{i+1}+x_{i}\right) / 2, i=1, \ldots n-1$ and $s_{n}=1$. For the members of the smoothing matrix $S=\left(s_{i j}\right)=\left(W_{i}\left(x_{j} ; h\right)\right), i, j=1, \ldots, n$, in the point of plan $x_{j}$ with the bandwidth $h$ for the GM estimate holds

$$
W_{i}\left(x_{j} ; h\right)=\frac{1}{h^{v+1}} \int_{s_{i-1}}^{s_{i}} K\left(\frac{x_{j}-u}{h}\right) d u .
$$

An advantage of the GM estimate is the possibility to estimate the function as well as its derivations.

The quality of the estimate is influenced by the value of the bandwidth to a great extent. If the bandwidth is small, the estimate is undersmoothed. If the bandwidth is large, the estimate is oversmoothed. The optimum bandwidth value can be estimated using several approaches. Nevertheless, the main aim is to minimize the average mean square error which can be expressed in the following form (Müller, 1988; Granovsky, Müller; 1991)

$$
\operatorname{AMSE}(\hat{m}, h)=\frac{1}{n} E \sum_{i=1}^{n}\left(m\left(x_{i}\right)-\hat{m}\left(x_{i} ; h\right)\right)^{2} .
$$


Let $K \in S_{v, k}$ There exist several estimates of this function which are asymptotically equivalent and unbiased (Härdle; 1990, Chiu; 1991). Most of them are based on the residual sum of squares $(R S S)$

$$
\operatorname{RSS}_{n}(h)=\frac{1}{n} \sum_{i=1}^{n}\left(Y_{i}-\widehat{m}\left(x_{i} ; h\right)\right)^{2} .
$$

As the type of the kernel function influences the quality of the resultant estimate, we will consider the optimum kernels, i.e. the kernels minimizing the AMSE (Granovsky, Müller, Pfeifer; 1995) (Gasser, Müller, Mammitzsch; 1985).

It is possible to use the cross-validation method for estimating the optimum bandwidth when working with real data (Hastie, Thibsirani, 1991; Drodge, 1994). The key idea of this method is to find a bandwidth corresponding to the minimum of the criterion of the cross-validation method. Such optimum bandwidth is denoted as $\widehat{h}_{o p t, G C V}$, the corresponding criterion can be written as follows

$$
\widehat{h}_{\text {opt }, G C V}=\arg \min _{h \in H} G C V(h),
$$

where $H$ is a set of bandwidth values for which the minimum of generalized crossvalidation is searched for. Usually, $H_{n}=\left[a_{k} n^{-1 /(2 k+1)}, b_{k} n^{-1 /(2 k+1)}\right]$ for some $0<a_{k}<b_{k}<\infty$. For the function $G C V$ the following holds

$$
G C V(h)=\frac{1}{n} \sum_{i=1}^{n}\left\{\frac{Y_{i}-\hat{m}\left(x_{i}\right)}{1-\operatorname{tr}(S) / n}\right\},
$$

where $\operatorname{tr}(S)$ denotes the sum of diagonal members of the smoothing matrix $S=\left(s_{i j}\right)=$ $\left(W_{i}\left(x_{j} ; h\right)\right), i, j=1, \ldots, n$, defined above (5).

An estimate of the optimum bandwidth in the case of the first derivative of the function $m$ can be obtained using the relation

$$
C V^{(1)}(h)=\frac{1}{n-1} \sum_{i=1}^{n-1}\left\{\Delta_{i}^{(1)}-\hat{m}_{-(i, i+1)}^{(1)}\left(x_{i}^{(1)}\right)\right\}^{2},
$$

where $x_{i}^{(0)}=x_{i}, \Delta_{i}^{(0)}=Y_{i}, x_{i}^{(1)}\left(x_{i+1}^{(0)}-x_{i}^{(0)}\right) / 2, \Delta_{i}^{(1)}=\left(\Delta_{i+1}^{(0)}-\Delta_{i}^{(0)}\right) /\left(x_{i+1}^{(0)}-x_{i}^{(0)}\right), i=1, \ldots, n$ and $\widehat{m}_{-(i, i+1)}^{(1)}\left(x_{i}^{(1)}\right)$ is the kernel GM estimate in the point $x_{i}^{(l)}$ constructed on the values $\left(x_{1}, Y_{1}\right), \ldots, \quad\left(x_{i-1}, Y_{i-1}\right),\left(x_{i+2}, Y_{i+2}\right), \ldots\left(x_{n}, Y_{n}\right)$. The number $\widehat{h}_{o p t, C V^{(1)}}$ minimizing the $C V^{(l)}$ (h) is the estimate of the optimum smoothing parameter for the estimate of the first derivation:

$$
\hat{h}_{o p t, C V^{(1)}}=\arg \min _{h \in \hat{H}_{n}} C V^{(1)}(h),
$$

where usually $\hat{H}_{n}=[1 / n, 2]$ (Drodge, 1994).

It can be shown (Horová, Zelinka; 2000) that the formula

$$
h_{o p t, v, k}^{*}=\left[\frac{(2 v+1) k}{k-v}\right]^{1 /(2 k+1)} h_{o p t, 0, k}^{*}
$$


holds for $v, k$ even and the formula

$$
h_{o p t, v, k}^{*}=\left[\frac{(2 v+1)(k-1)}{3(k-v)}\right]^{1 /(2 k+1)} h_{o p t, 1, k}^{*}
$$

holds for $v, k$ odd, where $h_{\text {opt }, 0, k}^{*}$ can be estimated using $\widehat{h}_{\text {opt }, G C V}$ and $h_{o p t, 1, k}^{*}$ can be estimated using $\hat{h}_{o p t, C V^{(1)}}$. It can be seen that if we deal with an estimate of the function derivation, it will be sufficient to estimate the bandwidth for $v=0$ (even) and $v=1$ (odd) according to the even or odd order of the derivation of the function which is estimated.

In the case of kernel estimates, the time axis $t=1, \ldots, n$ is transformed to equidistantly distributed design points $x_{i}$ in the interval $[0,1]$. This transformation will be used during the GM estimates calculations. For the identification of turning points according to the rules written in Canova (1999) the standard notation of time series $t$ will be used. Canova considered two standard mechanical dating rules. The first rule defines a trough as a situation where two consecutive quarters declines in the reference cycle are followed by an increase, i.e. $c_{t-2}>c_{t-1}>c_{t}<c_{t+1}$. Likewise a peak is defined by two consecutive increases followed by a decline, i.e. $c_{t-2}<c_{t-1}<c_{t}>c_{t+1}$. This right side variant will be completed by the left side version now. In this sense, a trough is defined as a situation where one quarter decline in the reference cycle is followed by two consecutive quarters of increase, i.e. $c_{t-1}>c_{t}<c_{t+1}<c_{t+2}$. Likewise, a peak is defined by one quarter increase followed by two consecutive declines, i.e. $c_{t-2}<c_{t-1}<c_{t}>c_{t+1}$, Bonenkamp (2001). The second rule selects a quarter as a trough (peak) if there are at least two consecutive negative (positive) spells in the cycle over a three-quarter period. The reason for applying both rules results from the tendency to minimize the risk of an early identification of turning points. The evaluation of existing turning points will be done on the basis of interconnecting the naive rules as written in Canova (1999) and Bonenkamp (2001) and a detailed analysis of the graphical representation of the growth business cycle behaving.

\section{Algorithm of Alternative Approach}

This alternative approach is based on mathematical principle of finding extremes by using nonparametric estimates (the Gasser-Müller estimates) of the time trend and its derivatives; in the context of business cycle analysis these extremes are called turning points. The method can be briefly described in the following several steps:

Step 1: Optimizing the bandwidth $h$ to obtain the estimate of the time trend $\hat{m}(x)$ of given data according to the formula (6), $\widehat{h}_{\text {opt }, G C V}$.

Step 2: Estimating the time trend $\hat{m}(x)$ according to the formula (4) for $v=0$ with the optimum bandwidth found in Step 1.

Step 3: Optimizing the bandwidth $h$ to obtain the estimate of the first derivative of the time trend $\hat{m}^{(1)}(x)$ of given data according to the formula $(9), \widehat{h}_{\text {opt }, C V^{(1)}}$. 
Step 4: Estimating the first derivative $\hat{m}^{(1)}(x)$ of the time trend according to the formula (4) $(v=1)$ with the optimum bandwidth found in Step 3.

Step 5: The identification of potential stationary points - turning points, i.e. points $x_{i}, i=1, \ldots, k$, for which $\hat{m}^{(1)}(x)=0$. The number $k$ is the number of intersections of $\hat{m}^{(1)}(x)$ and the $x$-axis.

Step 6: Optimizing the bandwidth $h_{o p t, v, k}^{*}$ to obtain the estimate of the second derivative of the time trend $\hat{m}^{(2)}(x)$ of given data according to the formula (11).

Step 7: Estimating the second derivative $\hat{m}^{(2)}(x)$ of the time trend according to formula (4) $(v=2)$ with the optimum bandwidth found in Step 6.

Step 8: The identification of type of extreme, i.e. a decision whether $\hat{m}^{(2)}(x)>0$ (minimum) or $\hat{m}^{(2)}(x)<0$ (maximum).

For the evaluation of the algorithm described above, our own procedures in Matlab were used (Poměnková, 2005).

\section{Data}

For the long-term trend identification and the business cycle dating in the Czech Republic the measurements of four macroeconomic indicators have been used. Namely, the gross domestic product (GDP), the total industry excluding construction (industry) and the gross capital formation (GCF); the available data sets are quarterly values in the period 1996/Q1 - 2008/Q4. The last one, the final consumption expenditure (consumption) includes the quarterly values in the period 1995/Q1 - 2008Q4. All input values were sourced from the free Eurostat webpage and they are in the form of chainlinked volumes, the reference year 2000 and they were transformed into the natural logarithms (Figures 1a-4a). The selected time series were sourced from the national accounts system of the GDP composite indicator which can be considered a reference indicator of economic activity in empirical analyses. The author supposes a significant similarity between the analysed time series because all of them indicate economic activity.

\section{Empirical Analysis}

The growth business cycle concept and subsequently the dating process assume that its individual phases determine increases and decreases in economic growth (for example the OECD). We can obtain the economic activity growth using several ways (Bonenkamp, 2001) which are based on de-trending the mentioned indicators. The most widely used method is the first differences (FOD). The unobserved component model (UC), regression curves, the Hodrick-Precott (HP) filter with the parameter $\lambda=$ 1600 or the Baxter-King band pass filter are also widely used. 
De-trending using the Hodrick-Prescott filter will be done for selected economic indicators. Smoothing parameter $\lambda=1600$ was chosen (Hodrick, Prescott; 1980), (Kapounek, 2009). The reasons are the following. De-trending using standard methods like the FOD, the UC, regression curves or the Baxter-King are not suitable for all analyzed time series. In the case of the Baxter-King band-pass filter several observations of input time series are lost since the methodology works with a moving window. It is not suitable for the Czech Republic sample size. On the other hand, the assumption for the FOD procedure applications is that the trend component of analysed time series is a random walk with no drifts and that the cyclical component is stationary and both components are uncorrelated (Canova, 1998). The GDP data sets do not correspond with the condition of uncorrelated components. In the unobserved components model, the assumption that the growth component follows a random walk with a drift is not satisfied for the industry, the consumption and the GCF. It is not suitable to use the Gasser-Müller estimate for de-trending either. Edge effects do not vanish even after applying the method designed for solving this problem. The edge effect has a bigger variance at the end of sample size. It can be caused by the size of bandwidth $[x-h, x+h] \not \subset[0,1]$, thus the weight function cannot use all smoothing bandwidth. The correction of the edge effect can be done by using the reflective technique or using the edge kernels (Poměnková, 2005 a 2008). On the contrary the HP filter gives good results without the end sample problem. The initial smoothing parameter $\lambda=1600$ for the quarterly values recommended by Hodrick and Prescot (1980) will be used for the empirical analysis.

The empirical analysis will be the same for all selected indicators and will be the following. Firstly, the Kendal $\tau$ - test and the Augmented Dickey-Fuller stationarity test (the ADF) will be applied. Consequently, the identification of turning points using the Gasser-Müller estimate will proceed. For this purpose the bandwidth estimate using several criteria will be investigated because the available sample size is $n<70$. Also the edge effects of estimates will be reviewed. Secondly, estimates of the first and the second derivation of the time series trend will be calculated. Using them the stationary points will be identified and expected troughs and peaks of business cycle will be specified. The corresponding confidence intervals will be incorporated for the estimated trends (Härdle, 1990). A precise determination of turning point moments requires interpolation. With respect to the quarterly data frequency the author decides to determine the moment of the turning point according to the data character.

In the process of the optimum bandwidth searching, the minimizing AMSE, namely the Akaik information criterion, the cross-validation method, the Rice penalization function, and the Fourier transformation method will be investigated (Härdle, 1990; Rice, 1984). On the basis of the estimated values a graphical representation and a resultant bandwidth estimate will be chosen. The class of optimum kernels will be used during this process. Notice that all results will be interpreted with respect to the small sample size. The obtained results of turning points will be compared to the naive rules as Canova (1998) or Bonenkamp (2001) wrote and the similarity will be judged.

According to the Kendal $\tau$-test and the ADF test we can say that all input values into the dating process of growth business cycle on the basis of total industrial production (industry), gross capital formation (GCF), consumption expenditure (consumption) and final gross domestic product (GDP) measurements de-trended by the Hodrick- 
Prescott filter are random. The GDP indicator is taken as the main reference indicator which, with respect to the summary character, can be distorted by exogenous shocks which partial indicators do not include.

The optimization of the bandwidth for the GM estimate was done; the results are shown in Table 1 below.

Table 1

Optimum Bandwidth Corresponding to the Kernel Type and Estimated Time Trend

\begin{tabular}{|l|c|c|}
\hline Estimated trend of & Bandwidth & Kernel \\
\hline Industry & $\mathrm{h}=0,23$ & $\mathrm{~S}_{0,12}^{1}$ \\
\hline 1st derivation; Industry & $\mathrm{h}=0,10$ & $\mathrm{~S}_{1,51}^{1}$ \\
\hline 2nd derivation; Industry & $\mathrm{h}=0,07$ & $\mathrm{~S}_{2,4}^{1}$ \\
\hline GCF & $\mathrm{h}=0,14$ & $\mathrm{~S}_{0,6}^{1}$ \\
\hline 1st derivation; GCF & $\mathrm{h}=0,12$ & $\mathrm{~S}_{1,5}^{1}$ \\
\hline 2nd derivation; GCF & $\mathrm{h}=0,15$ & $\mathrm{~S}_{2,6}^{1}$ \\
\hline Consumption & $\mathrm{h}=0,35$ & $\mathrm{~S}_{0,14}^{1}$ \\
\hline 1st derivation; Consumption & $\mathrm{h}=0,30$ & $\mathrm{~S}_{1,11}^{1}$ \\
\hline 2nd derivation; Consumption & $\mathrm{h}=0,35$ & $\mathrm{~S}_{2,14}^{1}$ \\
\hline HDP & $\mathrm{h}=0,23$ & $\mathrm{~S}_{0,12}^{1}$ \\
\hline 1st derivation; HDP & $\mathrm{h}=0,12$ & $\mathrm{~S}_{1,5}^{1}$ \\
\hline 2nd derivation; HDP & $\mathrm{h}=0,22$ & $\mathrm{~S}_{2,12}^{1}$ \\
\hline
\end{tabular}

Source: Own calculation.

\subsection{Industry}

On the basis of the first derivation estimate (Figure 1c) and the second derivation estimate (Figure 1d) of time trend and their confidence intervals, the following turning points for industry were identified (Table 2.)

Table 2

Expected Moments of Trough and Peak of the Czech Republic Growth Business Cycle Identified with the Help of GM Estimates (industry)

\begin{tabular}{|l|l|l|l|l|}
\hline Trough & $1998 / 3-1998 / 4$ & $2003 / 2-2003 / 4$ & $2007 / 2-2007 / 4$ & $2007 / 2-2007 / 3$ \\
\hline Peak & $1996 / 4-1997 / 1$ & $2000 / 2-2000 / 3$ & $2006 / 2-2006 / 3$ & $2007 / 3-2007 / 4$ \\
\hline
\end{tabular}

Source: Own calculation.

At first the evaluation of the calculated edge points is done. According to the figure (Figure 1b) we can see a more decreasing tendency of end points compared to the starting period. It can be supposed that this fact is caused by the end sample problem of HP filtering. Considering the GM estimate trend of this cycle, we do not identify any significant edge effects. At the beginning of design points we can see a potential peak after the expansion, the potential peak is followed by the recession at the end. 
Figure 1

Industry
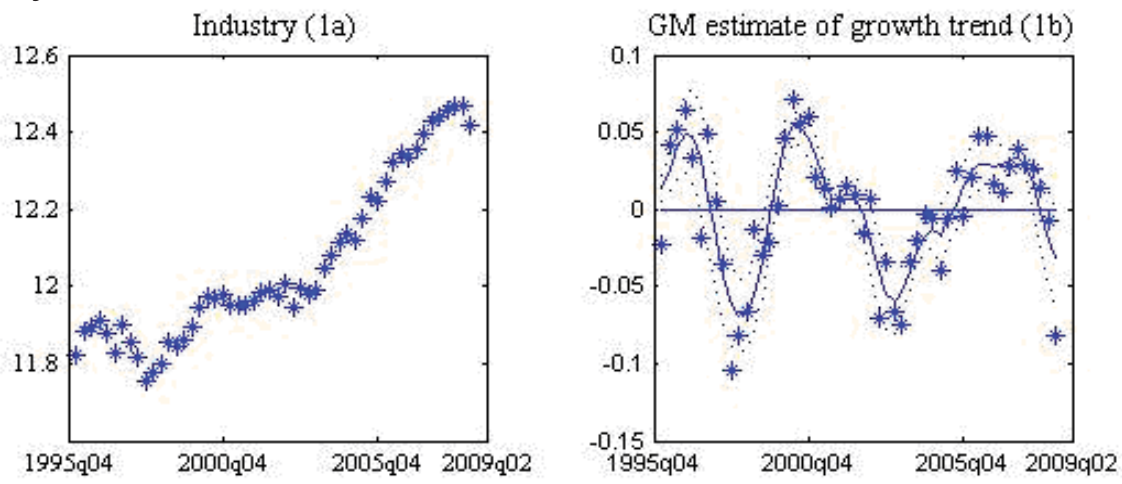

GM estimate of the 1 st derivation of growth trend (1c)

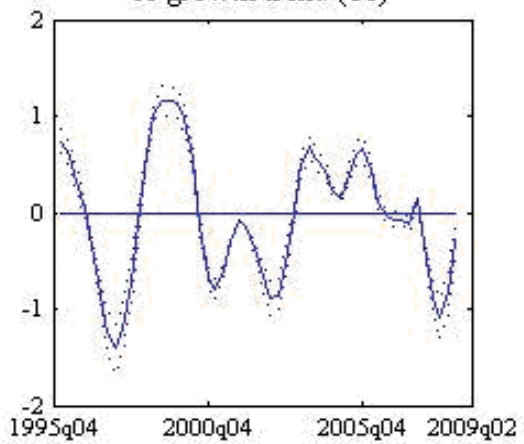

GM estimate of the 2nd derivation of growth trend (1d)

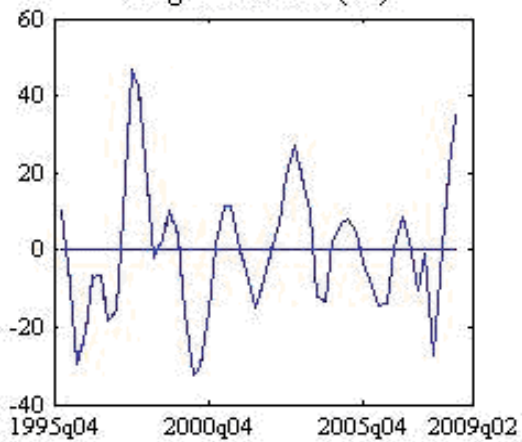

Source: Own calculation.

Let us focus on the last potential trough in 2007/2-2007/3 and peak in 2007/3$2007 / 4$. The values of GM estimate are in turn negative, positive and negative; in the period before 2007/2 until the previous extreme there appear only positive values, after 2007/4 until the end of the data set there appear negative values. With respect to the second order derivation estimate of time trend we can see small positive values in $2007 / 2$, strong negative values in 2007/3 and very small negative values in 2007/4. After 2007/4 until the end of the period strong negative values are followed by strong positive values. We can conclude that the minimum appears in 2007/2 and the maximum appears in 2007/3.

If we interconnect the graphic representation with the exact statistic methodological approach, we have to judge the sign of the estimated trend of industrial production growth. We find no sign changes there and thus the extremes - minimum and maximum - are spurious. Before 2005/3 economic activity is above its long-term trend. Focusing on the time trend estimate values of this business cycle indicator in 2007/2 - 2007/4 we can consider these figures very close and the economy in this time can be denoted rather as stagnating above the long-term trend of economic activity. Thus, the dating of the growth business cycle expansion and recession are not influenced by these spurious extremes. 
Using the naive rules (Canova, 1998) for identifying extremes we can see that the first rule identifies, apart from others, the minimum in $2007 / 1$ and the maximum in 2007/3. However, after the second rule application we arrive at the conclusion that these turning points are spurious. That is, the second rule works with the same theoretical background which we used in the GM estimate application. The year 2007 can be, therefore, considered a fluctuation period followed by a decrease of rate of growth at the end. The comparison of results obtained by these two approaches is given in Table 3 .

Table 3

Comparison of the Turning Points Identification in the Czech Growth Business Cycle (Industry)

\begin{tabular}{|c|c|c|c|}
\hline \multicolumn{2}{|c|}{ GM estimate } & \multicolumn{2}{c|}{ Naive rules } \\
\hline Trough & Peak & Trough & Peak \\
\hline & $1996 / 4$ & & $1996 / 4$ \\
\hline $1998 / 3$ & & $1998 / 2$ & \\
\hline & $2000 / 2$ & & $2000 / 2$ \\
\hline $2003 / 3$ & & $2003 / 4$ & \\
\hline & $2006 / 3$ & & $2006 / 2$ \\
\hline
\end{tabular}

Source: Own calculation.

As we can see in Table 3, the identified extremes have one quarter difference in three periods, in 1998, 2003 and 2006. A more detailed analysis of input values to the dating process for 1998/2 and 1998/3, 2003/3 and 2003/4 shows that we can consider the de-trended values and the GM estimates to be the same. Thus the growth business cycle in this time can be denoted as stagnation. The troughs identified by the GM estimate can be compared to the measurements established in 1998/3, 2003/3 and 2006/3.

We can conclude that both methods identify the same extremes and identically point out the year 2007 as questionable. In order to evaluate the results profoundly the graphic representation and the analyst's experience are needed.

\subsection{Gross capital formation}

In case of gross capital formation (GCF) the evaluation of edge points shows (Figure 2) that the growth business cycle trend estimate started and ended approximately at the same time. We can, therefore, suppose that using the HP filter for de-trending does not cause the end sample problem. According to the GM estimates result (the 1st and 2nd derivation time trend estimate) (Figure $2 b-2 c$ ) we can deduce the following potential moments for troughs and peaks (Table 4).

Table 4

Expected Moments of Trough and Peak of the Czech Republic Growth Business Cycle Identified with the Help of GM Estimates (GCF)

\begin{tabular}{|l|c|c|c|c|c|c|}
\hline Trough & $1997 / 4-1998 / 1$ & $1999 / 2-1999 / 3$ & $2003 / 2-2003 / 3$ & $2001 / 3-2001 / 4$ & $2005 / 2-2005 / 3$ & $2008 / 3-20084$ \\
\hline Peak & $1996 / 3-1996 / 4$ & $1998 / 2-1998-3$ & $2000 / 4-2001 / 1$ & $2001 / 4-2002 / 1$ & $2004 / 1-2004 / 2$ & $2007 / 1-2007 / 2$ \\
\hline
\end{tabular}

Source: Own calculation. 
Figure 2

Gross Capital Formation (GCF)
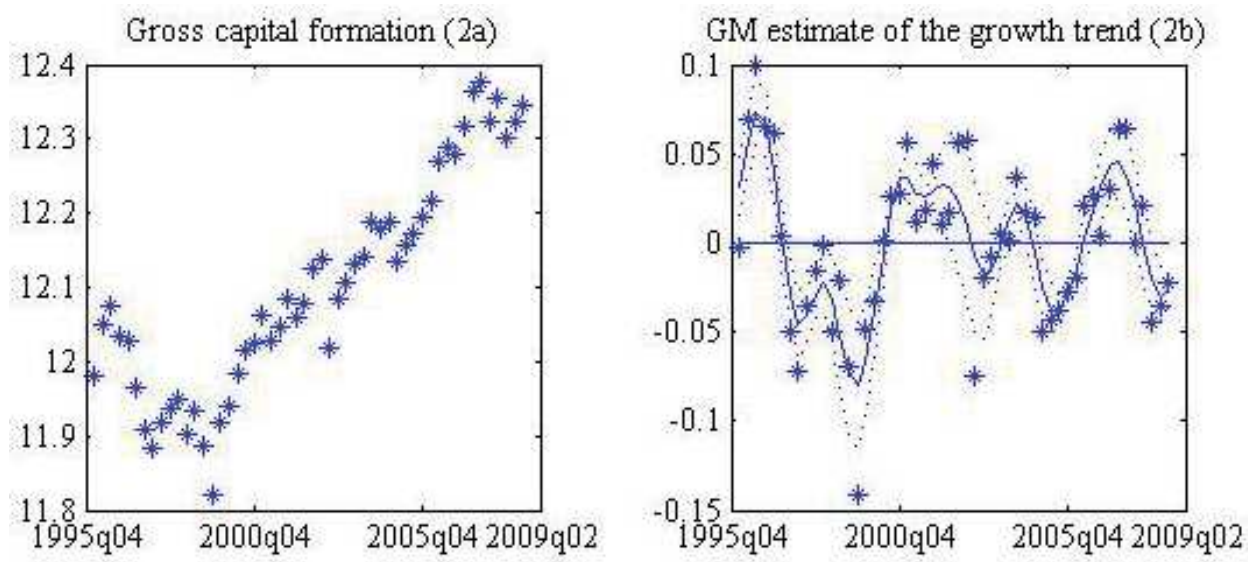

GM estimate of the 1st derivation of growth trend (2c)

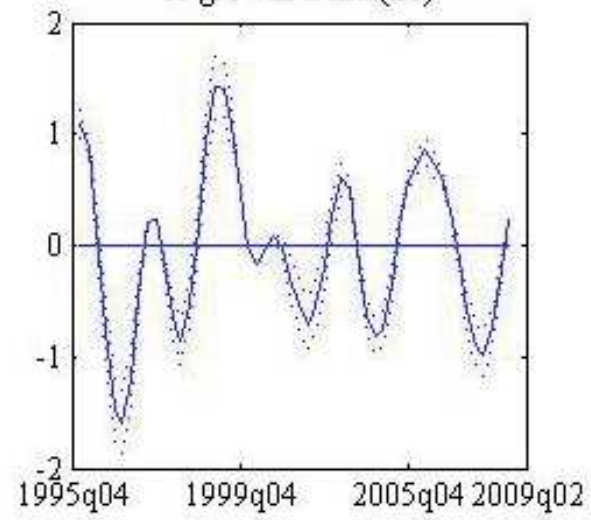

GM estimate of the 2 nd derivation of growth trend (2d)

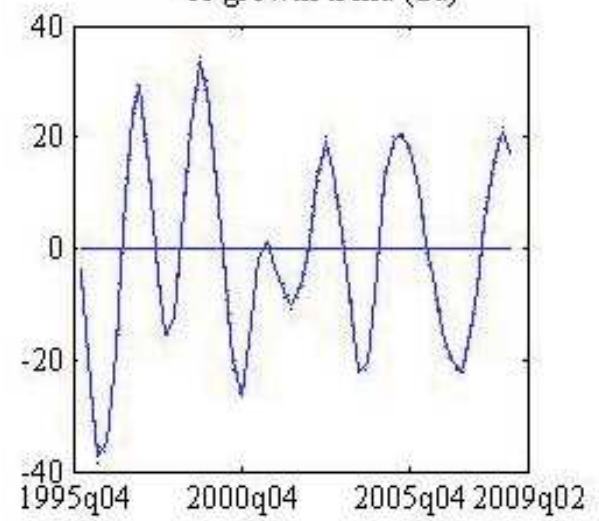

Source: Own calculation.

Like in the case of the total industry growth business cycle, the gross capital formation business cycle shows an ambiguous period; the period of 2001-2002. The values of the GM first derivation estimate are in turn negative (2001/3), positive (2001/4) and negative (2002/1). If we analyse the GM estimate of the second derivation we can identify the minimum in $2001 / 3$ and the maximum in 2001/4. This situation is similar to the situation for the industry in 2007/2 - 2007/4. Thus we again combine the graphic representation with the statistic approach with the aim to judge the sign of the estimated gross capital formation growth. Based on this analysis it can be stated that there are no changes and thus the indicated extremes - minimum and maximum - are spurious. In 2000/3 there were extremes which indicate a weak fluctuation above the economic activity long-term trend. Hence, the dating of expansion and recession are not influenced by these spurious extremes. 
The identification of extremes using the naive rules (Canova, 1998) for the period $2000 / 2-2002 / 4$ (the growth business cycle has positive values) shows that according to the first rule the whole range of extremes can be identified. However, after applying the second rule for the period 2000/2 - 2002/4 we can determine that except the extreme in 2001/1 denoted as the maximum the rest of extremes is spurious. The time trend evolution in 2000/2 - 2001/1 can be denoted as stagnation with slow decreases and increases above the long-term trend.

If the naive rules are strictly kept, the potential peak in 1998/3 will be denoted as spurious and rejected. The value in 1998/3 is negative and very small (- 0.0014). Regarding this, it does not satisfy the second naive rule in the sense that the value of extreme is greater than zero. However, if the total time series trend tendency of growth business cycle is evaluated and if we consider the fact that the growth business cycle values are obtained using the de-trending technique from the input values, we have to admit the existence of confidence interval where the growth business cycle values belong to. The confidence interval allows the estimated growth business cycle values to be more or less greater or smaller. Therefore, we cannot exclude the possibility that during the evaluation process in 1999/3 this moment will be denoted as peak and because of that together with the condition of alternation turning points, the moment $1997 / 4$ will be denoted as trough. Let us observe that this idea is supported by the result of the Bry-Boschan algorithm application (Bry, Boschan; 1971). This algorithm proved the moment 1997/4 as trought and the moment 1998/3 as peak. The estimate of growth business cycle in 2001/Q1 (0.0563) a 2002/Q4 (0.0567) causes that the naive rules denote the moment 2002/Q4 as peak. However, as the difference between the estimated values in the moment 2001/Q1 (0.0563) and 2002/Q4 (0.0567) is very small, nearly zero, we can conclude that the peak came earlier, in 2001/1.

The comparison of results obtained by these two approaches is written in Table 5 .

Table 5

Comparison of Turning Points Identification of CZ Growth Business Cycle (GCF)

\begin{tabular}{|c|c|c|c|}
\hline \multicolumn{2}{|c|}{ GM estimate } & \multicolumn{2}{c|}{ Naive rules } \\
\hline Trough & Peak & Trough & Peak \\
\hline & $1996 / 3$ & & $1996 / 3$ \\
\hline $1997 / 4$ & & $1997 / 4$ & \\
\hline & $1998 / 3$ & & $1998 / 3$ \\
\hline $1999 / 3$ & & $1993 / 3$ & \\
\hline & $2001 / 1$ & & $2001 / 1$ \\
\hline $\mathbf{2 0 0 3 / 2}$ & & $2003 / \mathbf{1}$ & \\
\hline & $2004 / 2$ & & $2004 / 2$ \\
\hline $\mathbf{2 0 0 5 / 2}$ & & $\mathbf{2 0 0 5 / 1}$ & \\
\hline & $2007 / 2$ & & $2007 / 2$ \\
\hline $\mathbf{2 0 0 8 / 3}$ & & $\mathbf{2 0 0 8 / 2}$ & \\
\hline
\end{tabular}

Source: Own calculation. 
Table 5 above shows that there is one quarter difference in three cases of the identified extremes. In more details we realise that in 2005/1 and 2005/2, 2008/2 and $2008 / 3$ the de-trended values as well as the GM estimates are the same. This cannot be stated in the case of 2003/1 and 2003/2 troughs. With respect to the discrete character of input values it is not possible to determine the time of trough more precisely. As specifying the turning points depends on the de-trending technique, one solution for the turning points specification is to identify turning points on the basis of several de-trending methods applied on one input time series (for the robustness of results) and subsequently to make the dating of turning points using the GM estimates and naive rules. Even though both approaches identify the same trough in $2008 / 3$, this trough will not be considered because the next phase is not unambiguously expansive.

By comparing both approaches we can conclude that except the trough in 2003 the same turning points were identified. The trough in 2003 was found by the GM estimate in the second quarter while the naive rules identified it in the first quarter of the year 2003.

\subsection{Consumption}

Figure 3

\section{Consumption}
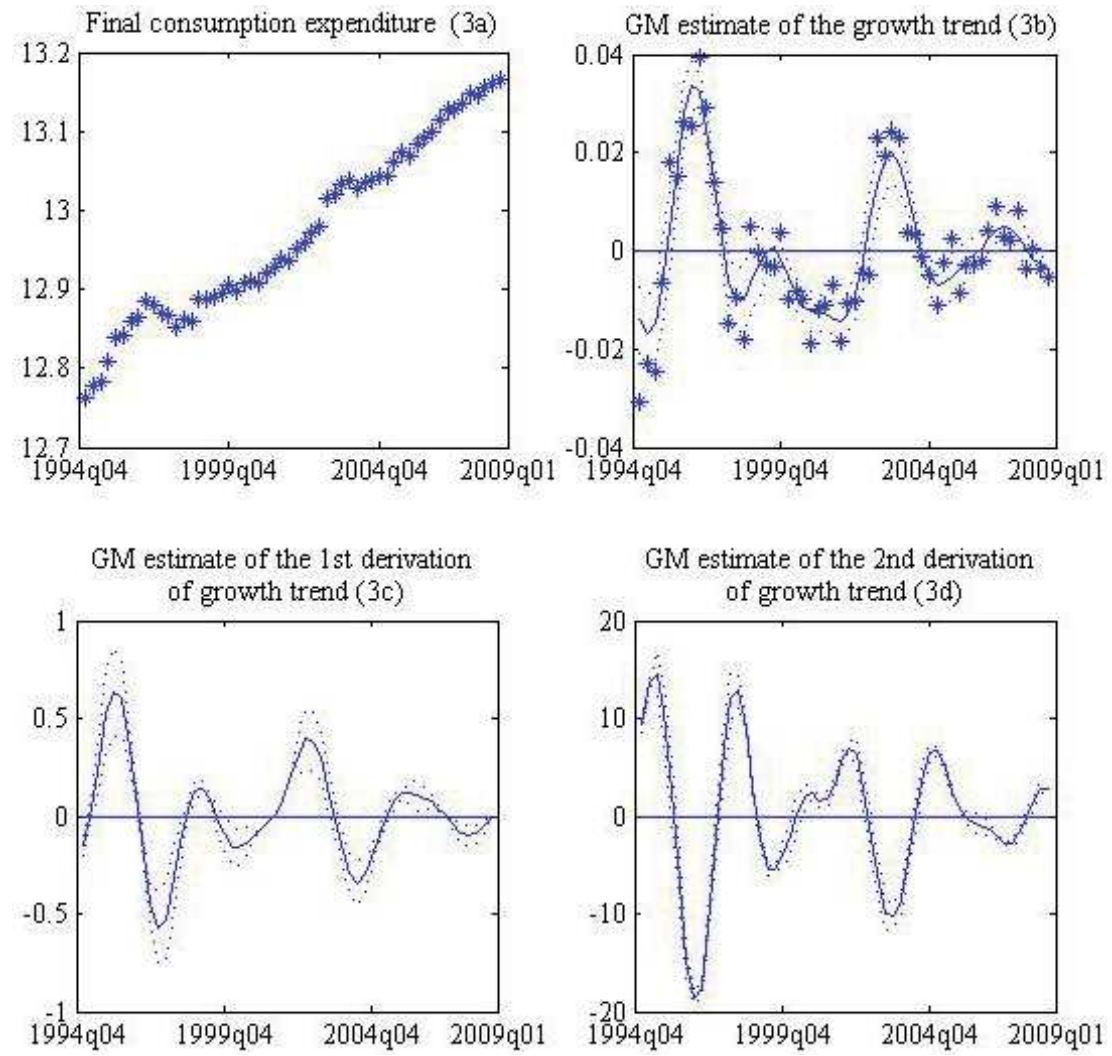

Source: Own calculation. 
The visual analysis of growth business cycle based on the final consumption expenditure (consumption) (Figure $3 b$ ) shows a strong value increase from the beginning of analysed period. We can permit the existence of the end sample problem caused by the HP filter de-trending. Considering the GM estimate of the cyclical time trend we cannot see any strong edge effects and thus the GM estimate of the time trend can be taken as sufficient to the input values. Comparing the growth business cycle values obtained by the HP filter de-trending with other de-trending techniques like the first order difference or regression (Bonenkamp, 2001) we can see a similarity among the character of values, without any major edge effects. Using the GM estimates results (the 1st and 2nd derivation time trend estimate) (Figure $3 b-3 c$ ) we can determine the following potential moments for the troughs and peaks (Table 6). The comparison of results obtained by these two approaches is written in Table 7 .

Table 6

Expected Moments of Trough and Peak of the Czech Republic Growth Business Cycle Identified with the Help of GM Estimates (Consumption)

\begin{tabular}{|l|c|c|c|c|}
\hline Trough & $1995 / 1-1995 / 2$ & $1998 / 2-1998 / 3$ & $2001 / 2-2001 / 3$ & $2005 / 1-2005 / 2$ \\
\hline Peak & $1996 / 4-1997 / 1$ & $1999 / 2-1999 / 3$ & $2003 / 1-2003 / 2$ & $2007 / 2-2007 / 3$ \\
\hline
\end{tabular}

Source: Own calculation.

If the naive rules are kept strictly, the potential extreme of peak in 1998/1 will be denoted as spurious and rejected. The identification of this moment as a real trough depends on the identification of the moment $1998 / 3$ as peak. The value in 1998/Q3 is very small and positive (0.041). To be a peak, the naive rules demand a limited number of small values from both sides around the potential turning point. This is not satisfied. However, if the total time series trend tendency of growth business cycle is evaluated, the naive rules can be marked as ineffective as they work only with a small number of values closed to the potential turning point. Since the growth business cycle values are obtained using a de-trending technique from the input values, the quality of obtained values describing the growth business cycle depends on the trend elimination. Then we have to admit the existence of a confidence interval where the growth business cycle values belong to. The confidence interval ensures that the estimated growth business cycle values can be more or less greater or smaller. Therefore we cannot exclude that during the evaluation process especially in 1999/1 this moment will be denoted as peak and because of that and under the influence of the condition of turning points alternation, the moment $1998 / 3$ will be denoted as trough.

From Table 7 we can see four different cases of turning point time identification which differ approximately one or two quarters. In more details we realise that in 1998/1 and 1998/2, 1999/2 and 1999/3,2003/2 and 2003/3, the values can be considered the same, i.e. both the de-trended values and the GM estimates. The values of consumption growth in 2007/1 and 2007/2 are different; however, the difference is very small. If we turn our attention to the GM trend estimate at this time we can see stagnation. Thus, the peak is classified in 2007/1. The trough 1995/1 will not be considered. Theoretically, with respect to the expansion phase of business cycle, this trough can be considered; however, determining the extreme time on the basis of available values can be questionable. 
Table 7

Comparison of the Turning Point Identification of the CZ Growth Business Cycle (Consumption)

\begin{tabular}{|c|c|c|c|}
\hline \multicolumn{2}{|c|}{ GM estimate } & \multicolumn{2}{c|}{ Naive rules } \\
\hline Trough & Peak & Trough & Peak \\
\hline $1995 / 1$ & & & \\
\hline & $1997 / 1$ & & $1997 / 1$ \\
\hline $1998 / 2$ & & $1998 / 1$ & \\
\hline & $1999 / 3$ & & $1999 / 4$ \\
\hline $2001 / 2$ & & $2000 / 4$ & \\
\hline & $2003 / 2$ & & $2003 / 3$ \\
\hline $2005 / 1$ & & $2005 / 1$ & \\
\hline & $2007 / 2$ & & $2007 / 1$ \\
\hline
\end{tabular}

Source: Own calculation.

In the case of values for 2000/4 and 2001/2 we cannot observe the agreement of identified times in both approaches. Indeed, the GM estimate shows a stagnancy with a slowly decreasing tendency in the period 2000/3 - 2001/2, but the de-trended values break this tendency in 2000/4. Thus, the strong decrease of values in 2000/4 in the case of naive rules application according to Canova determine just this value as trough, while the GM approach states the trough in a later period. The author is inclined to assume that the right trough is identified using the GM estimates in 2001/2, also for the fact that after the breaking point in 2000/4 the values come back to their previous tendency of decreasing trend.

By comparison of both approaches we can conclude that except one extreme the same turning points were identified. In the case of the polemic extreme, the trough in $2001 / 2$ or $2001 / 4$, the author inclines to the result obtained by using the GM estimates, i.e. in $2001 / 4$.

\subsection{GDP}

On the basis of GM estimate of the first (Figure 4c) and the second (Figure 4d) time trend derivation of growth business cycle based on the GDP and their confidence intervals the stationary points were determined. Thus, the decision of potential extremes - maximum and minimum - was done. The expected moments for turning points were deduced from the results (Table 8). The precise determination of turning point moments would require interpolation. With respect to the quarterly data frequency, we decide according to the graphic representation.

At first, the evaluation for the end sample points was done. If we compare the input values (Figure 4a), we can state that the estimate of the GDP growth trend begins and ends approximately in the same level. We can, therefore, suppose that the HP filter de-trending does not cause edge effects. The values of growth business cycle at the end of the analysed period reach bigger negative values compared to the values at the beginning of the sample size. Nevertheless, we suppose that as the cycle phase is in the 
recession without a possibility to identify a potential trough (the lack of data) we do not expect a significant impact on the last potential extreme, in our case a peak.

Figure 4

GDP
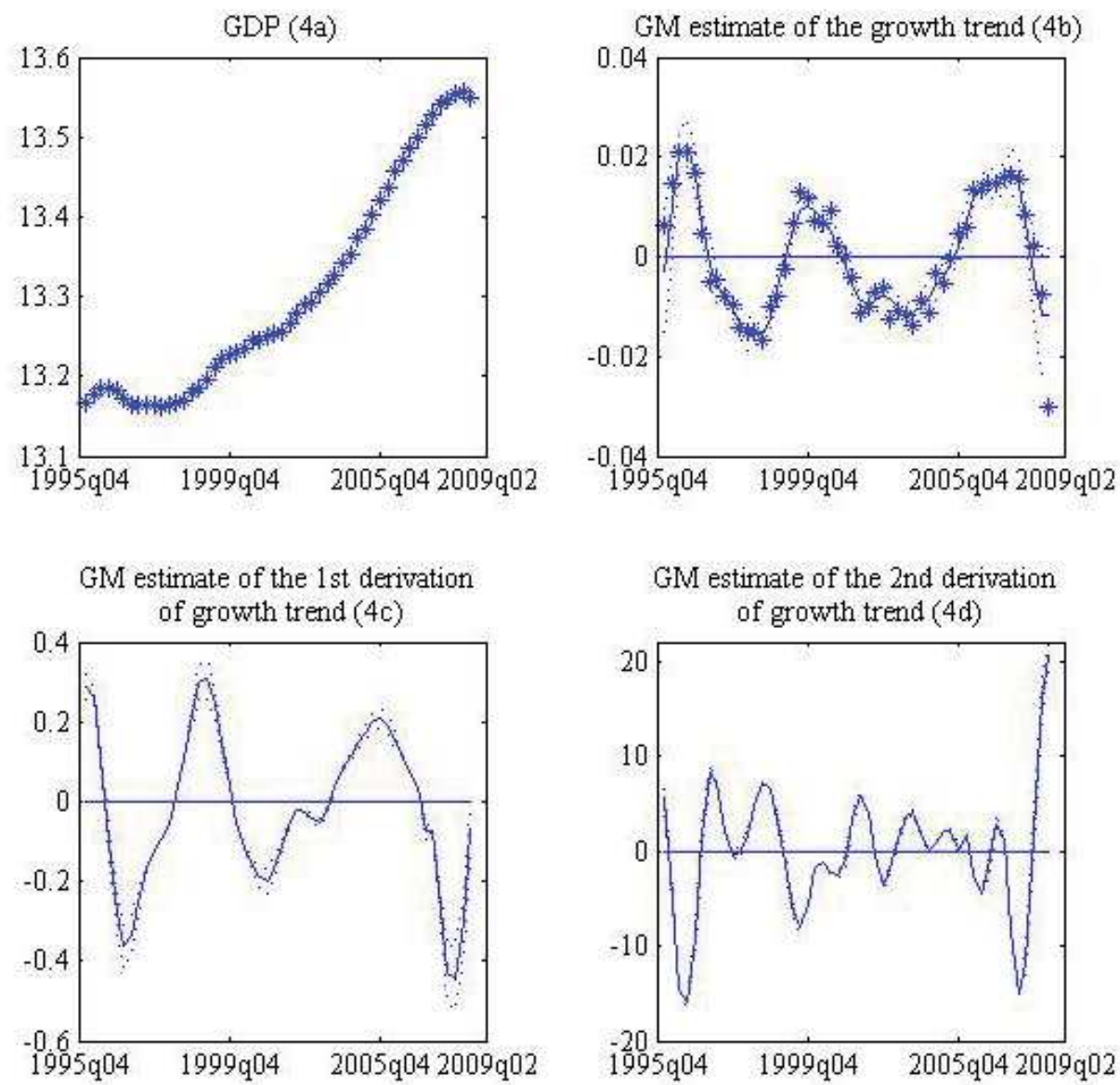

GM estimate of the 2 nd derivation of growth trend (4d)

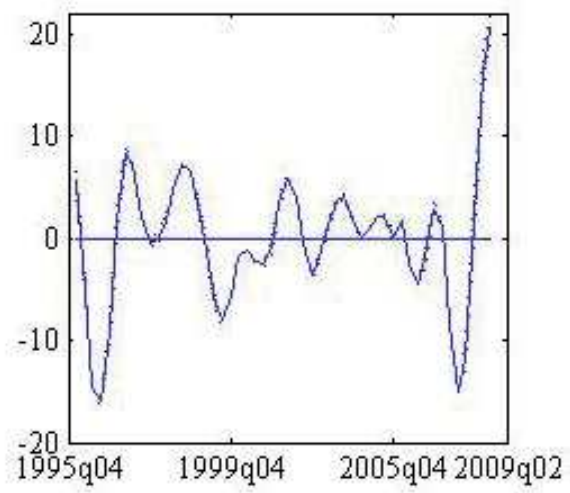

Source: own calculation

Here, a situation similar to the consumption arises. Comparing the GDP growth values obtained by the HP filter de-trending to other de-trending techniques like the first order difference or regression (Bonenkamp, 2001) we can see a strong similarity among the character of values without any major edge effects. Using the GM estimates results (the 1 st and 2 nd derivation time trend estimates) (Figure $4 \mathrm{~b}-4 \mathrm{c}$ ) we can arrive at the following potential moments for troughs and peaks (Table 8). The comparison of results obtained by these two approaches is given in Table 9 . 
Table 8

Expected Moments of Troughs and Peaks of the Czech Republic Growth Business Cycle Identified with the Help of GM Estimates (GDP)

\begin{tabular}{|l|c|c|c|}
\hline Trough & $1998 / 4-199 / 1$ & $2004 / 1-2004 / 2$ & \\
\hline Peak & $1996 / 3-1996 / 4$ & $2000 / 4-2001 / 1$ & $2007 / 2-2007 / 3$ \\
\hline
\end{tabular}

Source: Own calculation.

Table 9

Comparison of the Turning Point Identification of the CZ Growth Business Cycle (GDP)

\begin{tabular}{|c|c|c|c|}
\hline \multicolumn{2}{|c|}{ GM estimate } & \multicolumn{2}{c|}{ Naive rules } \\
\hline Trough & Peak & Trough & Peak \\
\hline & $1996 / 4$ & & $1996 / 4$ \\
\hline $1998 / 4$ & & $1998 / 4$ & \\
\hline & $2000 / 4$ & & $2000 / 3$ \\
\hline $2004 / 2$ & & $2004 / 2$ & \\
\hline & $2007 / 3$ & & $2007 / 3$ \\
\hline
\end{tabular}

Source: Own calculation.

Table 8 shows the same moments for identified turning points except one - in the year 2000 for the growth business cycle represented by the GDP values. A more detailed analysis of input values for 2000/3 and 2000/4 shows that both the de-trended and GM estimated values can be considered the same. Let us remark that this similarity is present also in the case of 2006/3 and 2006/4. The growth business cycle in these two quarters can be denoted as stagnant and the peak identified using the GM approach and compared to the empirical values can be established between 2000/3 - 2000/4. We can conclude that both approaches give the same results and can be taken as comparable from this point of view.

\section{Results}

On the basis of GM approach, the growth business cycle dating of the Czech Republic was done. The results of the presented methodological approach are demonstrated on the real values of the gross domestic products, the total industry excluding construction, the gross capital formation (the investment) and the final consumption expenditure sourced from the national accounts system and they are given in Table 10 below.

The GDP cyclical movements are closely connected to the industrial production (about 1/3 share of GDP). The identified peaks and troughs correspond to minimal lags, 1 quarter in the year 1998 and 2 quarters in the year 2000. The peaks and troughs of the investment (the gross capital formation) are often considered to predict the business activity. This fact is confirmed by the peaks in 1996 and 2007 and the trough in 2003 (Table 10). The consumption does not confirm any significant relationship to the turning points of GDP in the Czech Republic. However, the GM estimation approach allows us to identify peaks and troughs precisely not only in business cycle 
movements (represented by the GDP) but also in other indicators of economic activity fluctuation.

Table 10

Comparison of the Business Cycle Turning Points for Different Input Values

\begin{tabular}{|c|c|c|c|c|}
\hline time & Consumption & GCF & Industry & GDP \\
\hline $1996 q 03$ & & P & & \\
\hline 1996q04 & & & $\mathrm{P}$ & $\mathrm{P}$ \\
\hline 1997q01 & $P$ & & & \\
\hline 1997q04 & & T & & \\
\hline 1998q02 & T & & & \\
\hline 1998q03 & & $P$ & $\mathrm{~T}$ & \\
\hline 1998q04 & & & & $\mathrm{T}$ \\
\hline 1999q03 & $P$ & $\mathrm{~T}$ & & \\
\hline $2000 q 02$ & & & $P$ & \\
\hline $2000 q 04$ & & & & $P$ \\
\hline 2001q01 & & $P$ & & \\
\hline 2001q02 & $\mathrm{T}$ & & & \\
\hline $2003 q 01$ & & $\mathrm{~T}$ & & \\
\hline $2003 q 02$ & $P$ & $\mathrm{~T}$ & & \\
\hline $2003 q 03$ & & & $\mathrm{~T}$ & \\
\hline $2004 q 02$ & & $\mathrm{P}$ & & T \\
\hline $2005 q 01$ & $\mathrm{~T}$ & & & \\
\hline $2005 q 02$ & & $\mathrm{~T}$ & & \\
\hline $2006 q 03$ & & & $P$ & \\
\hline 2007q02 & $P$ & $P$ & & \\
\hline $2007 q 03$ & & & & $P$ \\
\hline
\end{tabular}

Source: Own calculation.

Statistical significance at the $1 \%\left({ }^{* * *}\right), 5 \%\left({ }^{* *}\right), 10 \%\left({ }^{*}\right), P($ peak), T (trough).

\section{Conclusion}

The presented paper provides the methodological background for an alternative approach in the business cycle dating process. The concept is based on the mathematical principle of identifying extreme points by using estimates of the time series trend derivations. For this purpose the nonparametric GM estimate is used. The obtained results are compared to the widely used dating technique mentioned in Canova (1998, 1999) or Bonenkamp (2001) which is denoted as the naive dating technique. The presented new method is taken as a specification method for the identification of turning points in the business cycle dating process. The following indicators of economic activity are used: the gross domestic product, the total industry excluding 
construction and the gross capital formation. The available data sets are the quarterly observations in the period 1996/Q1 - 2008/Q3. The last one, the final consumption expenditure (the consumption) is based on the quarterly values 1995/Q1 - 2008 Q4.

On the basis of empirical analysis we can conclude that the GM approach to the turning point identification process gives qualitatively precise results. The classified troughs and peaks in business cycle dating procedure on the basis of four economic indicators satisfy the economic assumptions for the cycle identification as Artis (2004) wrote. Troughs and peaks are alternating, the minimum duration for the phase is not shorter than two quarters and the cycle length is not shorter than five quarters.

Let us remark that the correct business cycle dating with the use of the naive rules required additional analyses (the graphical analysis and Bry-Boschan algorithm) in two cases, namely the gross capital formation and the final consumption expenditure. Thus, in case we apply the naive rules, there may appear situations when these rules are not effective as they do not consider the evaluation of time series tendency, but they work only with a small number of values close to the turning points.

The concept of turning point identification using the GM estimate considers all values of input time series in its construction while the naive rules work only with several consequent values. It can be, therefore, supposed that despite the mathematical character of this method it gives more precise results than the naive one. An advantage of naive rules application is their simplicity; however, this approach is not able to eliminate a possible noise in the data file caused for example by the data character, methodology of data collection or other mistakes. Also, the use of this method is time demanding in the case of large sample size. The procedure of the statistical approach represented by the GM estimates is more complicated; however, its application is more suitable for the cases of large sample size. Another advantage of this approach is its smaller sensibility to the data distribution assumption which does not take place in the case of nonparametric methods. Compared to the naive rules it is necessary to de-trend the data file at first. Commonly used de-trending techniques are mostly parametric, i.e. assumptions for the de-trending technique usually require the normal distribution.

In the context of GM estimate application some problems can arise. The bandwidth and the kernel order influence the quality of the estimate, and therefore both parameters are suitable to optimize. In the case of bandwidth several methods are suitable to use for the robustness of results. Further, because of the asymptotical character of kernel estimates, the sample size belongs to the next influencing factor. We can say that the resultant estimates are better for a bigger sample size than for a small sample size. A bigger sample size is usually a sample with more than 50-70 measurements. Another limitation of the application on real data is the assumption that the data file is random. Unfortunately macroeconomic data of transitive economy do not often satisfy this assumption. If, for practical reasons or from the economic theory point of view, it is not possible to make any data transformation leading to the satisfaction of random assumption, then it is necessary to keep this fact in mind in the case of parameter optimization and therefore to use several criteria. Especially in the case of autocorrelation in the data set the optimum bandwidth could cause the undersmoothing of the resultant estimate. Edge effects also belong to potential problems of the kernel estimates. The reflexion technique or edge kernels can be used for their correction (Poměnková, 2005 and 2008). 
With respect to the discrete character of input values, the GM approach can be taken as a specifying method for the business cycle dating. Its use offers a possibility to describe the long-term behaving of cyclical component in a more flexible way. The GM approach can take into consideration any observation during the construction of the estimation of any point and thereby produces not only a flexible but also a smooth estimate. In comparison with the naive technique it does not evaluate concrete de-trended values by mutual comparison of several values before and after the turning point, but it allows us to find the turning point in the context of all values. Thus it is suitable to connect the estimate based on the naive technique application with an exact mathematical approach like the GM approach. The naive technique is insufficient; more complicated dating rules may improve the quality of the outcomes as Canova wrote and as we proved. This methodology can contribute to the decision process of policy-makers, especially in stabilization policy implementation.

\section{References}

Artis, M., Marcellino, M., Proietti, T. (2004), "Characterising the Business Cycles for Accession Countries." CEPR-EABCN Conference of Business Cycle and Acceding Countries, Vienna, 2004.

Bonenkamp, J., Jacobs, J., Kuper, G. H. (2001), Measuring Business Cycles in the Netherlands, 1815-1913: A Comparison of Business Cycle Dating Methods. SOM Research Report, No. 01C25, Systems, Organisation and Management, Groningen. University of Groningen [online].

Canova, F. (1998), "De-trending and Business Cycle Facts." Journal of Monetary Economic, Vol. 41, pp. 533-540.

Canova, F. (1999), "Does De-trending Matter for the Determination of the Reference Cycle and Selection of Turning Points?" The Economic Journal, Vol. 109, No. 452 (January 1999), pp. 126-150.

Chiu S. T. (1991), "Some Stabilized Bandwidth Selectors for Nonparametric Regression." Annals of Statistics, 19, 1528-1546

Droge, B. (1994) "Some Comments on Cross-Validation." In Härdle, W., Schimek, M. G., eds., Statistical Theory and Computational Aspects of Smoothing. Austria, 1994, pp. 178-199.

Gasser, T. L., Müller, H. G., Mammitzsch, V. (1985), "Kernels for Nonparametrics Curve Estimation." J. Royal Statistical Society B47, pp. 238-251.

Granovsky, B. L. , Müller, H. G. (1991), "Optimizing Kernel Methods: A Unifying Variational Principle." International Statistical Review. 1991, 59, 3, pp. 373-388.

Granovsky, B. L., Müller, H. G., Pfeifer, C. (1995), "Some Remarks on Optimal Kernel Function." Statistics \& Decision 13, München 1995, pp. 101-116.

Hastie, T. J., Tibshirani, R. J. (1991), Generalized Additive Models. University of Toronto, Great Britain.

Härdle, W. (1990), Applied Nonparametric Regression. Cambridge University Press, 349 p.

Hodrick, R. J., Prescott, E. C. (1980), "Post-war U.S. Business Cycles: An Empirical Investigation." Carnegie-Mellou University, Pitsburgh, PA., 24 pp.

Horová, I. (2000), Some Remarks on Kernels, Journal of Computational Analysis, Vol. 2., No. 2, 2000 , pp. 253-263.

Horová, I. (2002), "Optimization Problems Connected with Kernel Estimates," Signal Processing, Communications and Computer Science. 2002 by World Scientific and Engineering Society Press, pp. 339-334.

Horová, I., Zelinka, J.. (2000), “Základy a aplikace jádrových odhadů. Analýza dat 2000/II." Moderní statistické metody, Lázně Bohdaneč, 21. - 24. 11. 2000, pp. 141-167. 
Kapounek, S. (2009), Estimation of the Business Cycles - Selected Methodological Problems of the Hodrick-Prescott Filter Application. Polish Journal of Environmental Studies. Vol. V, No. 6, p. 2. ISSN 1230-1485.

Poměnková, J. (2005), Some Aspects of Regression Function Smoothing (1st ed.). Ostrava, $\mathrm{PhD}$-thesis.

Poměnková, J. (2008), "Remarks to Optimum Kernels and Boundary Optimum Kernels." Applications of Mathematics. Vol. 53, No. 4, pp. 305-317.

Müller, H. G. (1988), „Nonparametric Regression Analysis of Longitudinal Data.“ Lecture Notes in Statistics 46. Springer-Verlag.

Rice, J. (1984), "Bandwidth Choice for Nonparametric Regression." The Annals of Statistics, 12, 1215-1230, 1984.

Wand, M. P., Jones, M. S. (1995), Kernel Smoothing (1st ed.). Chapman \& Hall, London.

\section{Webpages:}

http://epp.eurostat.ec.europa.eu/portal/page/portal/eurostat/home/ [18. 3. 2009] 